Academic Platform Journal of Engineering and Science

journal homepage: http://apjes.com/

\title{
Tongue-Operated Biosignal over EEG and Processing with Decision Tree and kNN
}

\author{
${ }^{* 1}$ Kutlucan Gorur, ${ }^{2} \mathrm{M}$. Recep Bozkurt, ${ }^{3} \mathrm{M}$. Serdar Bascil, ${ }^{1}$ Feyzullah Temurtas \\ ${ }^{1}$ Department of Electrical and Electronics Engineering, Bandirma Onyedi Eylul University, Turkey, \\ kgorur@bandirma.edu.tr, \\ temurtas@gmail.com, id \\ ${ }^{2}$ Department of Electrical and Electronics Engineering, Sakarya University, Turkey, \\ mbozkurt@sakarya.edu.tr, \\ ${ }^{3}$ Department of Biomedical Engineering, Selcuk University, Turkey, \\ serdarbascil@gmail.com,
}

Research Paper

Arrival Date: 03.07.2019

Accepted Date: 17.12.2020

\begin{abstract}
Tongue-machine interface (TMI) is a feasible way between the assistive technologies and paralyzed individuals who have lost their abilities to communicate with the environment. Researchers have presented equipment based tongue-machine interfaces to reach a reliable and speedy system. However, this kind of interfaces may occur a way of obtrusive, unattractive and unhygienic for disabled persons. In this research, we intended to propose a natural, unobtrusive and robust glossokinetic potential signals (GKP) based TMI exploring the success of the novel machine learning algorithms. The tongue is bound up with cranial nerves to the brain, which can escape from the spinal cord injuries in general. Moreover, the tongue has highly capable of sophisticated manipulation tasks with less perceived exertion in the oral cavity and gives degrees of privacy. In this study, ten naive healthy subjects have attended who were between 22-34 ages. Decision Tree (DT) and k-Nearest Neighbors (kNN) algorithms were used with Mean-Absolute Value (MAV) and Power Spectral Density (PSD) methods. Moreover, Discrete Wavelet Transform (DWT) was implemented to reveal the theta and delta subbands. In the study, the highest value was provided as $96.77 \%$ by the k-Nearest Neighbor algorithm for the best participant. Furthermore, the GKP-based TMI may be an alternative system for the limitations of the brain-computer interfaces. It is well-known that EEG deficits are major concerns for brain-computer interfaces.
\end{abstract}

Keywords: Glossokinetic Potential, Tongue-Machine Interface, Brain-Computer Interface, Decision Tree, k-Nearest Neighbors, Discrete Wavelet Transform

\section{INTRODUCTION}

Patients suffer from the loss of motor control because of the spinal cord injuries (SCIs), brain stroke or amyotrophic lateral sclerosis (ALS). These individuals do not use peripheral nerves and muscles in regular outputs. Assistive technologies (ATs) enable impaired persons to control and communicate a device such as computers, wheelchairs, neuroprosthesis or word-spellers [1]. Tongue-machine interface (TMI) is a tongue- managed system to operate assistive technologies by voluntary tongue motions. There is substantial research on a number of TMIs. According to recent reports, most of the systems operated by the tongue, have pieces of equipment in the mouth and around the headset [2]. These devices can disturb paralyzed people in their swallowing, breathing and speaking performances and they are not hygienic in the mouth [3,4]. In this study, we aimed to explore the performance of the Decision Tree and k-Nearest Neighbors machine learning algorithms in advancing a natural, reliable and easy-to-use 1-D control and communication tongue-machine interface using the glossokinetic potential signals (GKPs) solely. GKPs originate from a noncerebral region and occur low-frequency interference with the alpha and beta frequencies of brain activity. Because GKPs emerge generally in the delta (1-3 $\mathrm{Hz}$ ) and theta $(4-7 \mathrm{~Hz})$ bands. Then these rarely used signals are created by contacting the buccal walls with the tip of the tongue. If the tongue touches the buccal walls, the charge decreases in contact surface and vice versa operation created in the non-contact area [5].

The oral cavity is a sensitive region compared to other body parts. Hence, oral structures possess a cortex structure matching to hand size, whereas the lower limbs of the body possess a relatively small mapping in the somatic sensory cortex. Moreover, psychophysical papers on strength sensitivity and discrimination confirm that some mouth parts like the tongue are more susceptible than the fingertip [6]. For this reason, the tongue may perform encouraging results in a tongue-machine interface associated with 1-D pattern

${ }^{* 1}$ Corresponding Author: Bandirma Onyedi Eylul University Electrical- Electronics Engin. Depart. Bandirma-Balıkesir-Turkey/ kgorur@bandirma.edu.tr 
recognition compared to other body parts due to cortex brain mapping by measuring the signals over the scalp [7-9]. Inherent problems including degrees of freedom (DoF) and loss of control (LoC) are seen in in traditional braincomputer interfaces (BCIs). These problems are originated from the nonstationary electroencephalography (EEG) signals $[10,11]$. Furthermore, long training times and high cognitive efforts are drawbacks of BCIs $[12,13]$. GKP-based TMI can solve to these problems without so much energy because of the intuitive tongue motions.

Recently, few of the tongue-operated interfaces handled glossokinetic potential responses in the literature. Nam et al. have developed "Tongue-Rudder" [3]. In this research, glossokinetic potential responses were used for 1-D extraction and EMG signals of the jaw utilized for calibration and toggle operations to control a wheelchair. Linear variations of GKP were measured from the scalp and earlobes to be classified by principal component analysis (PCA). Then, the same research group has developed "GOM-face" [4]. In this work, GKP and EOG (Electrooculogram) were implemented for each 1-D extraction and EMG used for toggle and calibration settings again to manage a humanoid robot. Tongue and eyes are named as charged organs based on the same phenomena of occurring. Thus, all signals were measured over the face. Then, eigenvalue decomposition of two covariances was calculated to discriminate GKP and EOG signals to be classified by support vector machine algorithm. However, implementing Decision Tree and k-Nearest Neighbor algorithms with MAV and PSD techniques in GKP-based TMI is the first attempt to show the performance of these machine learning algorithms.

Primary of hardware-based tongue-driven systems are presented by Huo et al. [1]. They have developed a series of wireless tongue-operated interfaces with attaching a piece of permanent magnet on the tongue and placing sensors in the mouth or on the dental-set/headset to detect the magnetically induced changes [1, 14-16]. Then, transmitted data were processed by computer or smartphone. Krishnamurthy et al. handled the same phenomena to realize a TMI [17]. Nonetheless, this kind of systems might cause disabled persons uncomfortable and unhygienic access in daily life. However, our study, glossokinetic potential responses on a tongue-machine interface may offer handicapped people in a natural and easy-to-use control in assistive devices. The other design approach of a TMI by Vaidyanathan et al. is based on airflow pressure changes created by the tongue movements, therefore attaching a microphone to the air canal [18-22]. However, GKP-based TMI can handle to manage an AT without affecting listening performance due to the acquisition the signals over the scalp.

Three experimental paradigms were established in the offline recording system. Decision Tree and k-Nearest Neighbor algorithms were applied as MLs with Mean-Absolute Value and Power Spectral Density methods to recognize the pattern of GKPs on the scalp. Moreover, Discrete Wavelet
Transform (DWT), Principal Component Analysis (PCA), and Independent Component Analysis (ICA) were also utilized in preprocessing and feature selection operations respectively.

\section{MATERIALS AND METHODS}

In this study, the procedure of the spatial pattern classification is as follows: Acquisition signals were carried out over the scalp based on the 10-20 international electrode placement system [23]. Then preprocessing steps are implemented as; DWT is to extract the delta and theta frequency subbands of the signals. Then the raw data set is normalized. After that, feature extraction operation is applied to highlight the data set. Principal Component Analysis and Independent Component Analysis are to decrease the features' dimension of the same data set. Finally extracted data are conveyed to the classifiers. GKP occurence and GKP-based TMI workflow was presented in Fig.1.

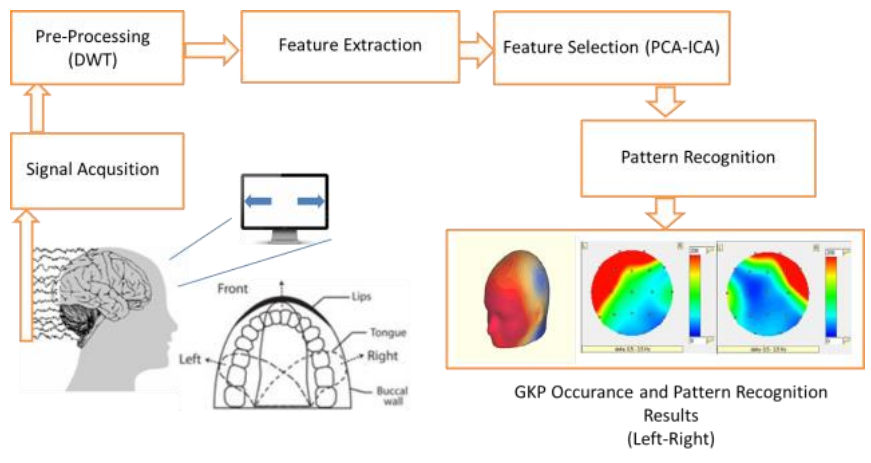

Fig.1. The presentation of GKP occurance and GKP-based tongue machine interface

GKP signals were collected by EEG measurement device using the Micromed SAM32RFO with 19 channels, and impedances of electrodes were kept under $10 \mathrm{k} \Omega$. In the acquisition system, Left (A1) and right (A2) earlobes and left-eyebrow were designated as reference points and ground respectively. Each channel was sampled at a frequency of $1024 \mathrm{~Hz}$ and filtered $(0.5-100 \mathrm{~Hz})$. Besides, a notch filter of $50 \mathrm{~Hz}$ was applied to remove the power line noise [24]. The electrodes for monopolar installation is represented in Table 1. Extracted data of delta and theta frequencies were normalized in the range of 0-1 according to the Eq.1.

$$
X_{S}^{\text {norm }}=\frac{X_{S}-X_{\min }}{X_{\max }-X_{\min }}
$$

$X_{S}$ stands for the sth data, $X_{\min }$ and $X_{\max }$ describe the minimum and maximum values in the data set, respectively [25]. This step helps ML algorithms to classify the data sets easier. 
Table 1. The Monopolar Placement for EEG Electrodes

\begin{tabular}{cc}
\hline $\begin{array}{c}\text { Number } \\
\text { of } \\
\text { Channels }\end{array}$ & $\begin{array}{c}\text { Names of } \\
\text { Channels }\end{array}$ \\
\hline Chnl-1 & Fp2 \\
\hline Chnl-2 & Fp1 \\
\hline Chnl-3 & F7 \\
\hline Chnl-4 & F3 \\
\hline Chnl-5 & Fz \\
\hline Chnl-6 & F4 \\
\hline Chnl-7 & F8 \\
\hline Chnl-8 & T3 \\
\hline Chnl-9 & C3 \\
\hline Chnl-10 & Cz \\
\hline Chnl-11 & C4 \\
\hline Chnl-12 & T4 \\
\hline Chnl-13 & T5 \\
\hline Chnl-14 & P3 \\
\hline Chnl-15 & Pz \\
\hline Chnl-16 & P4 \\
\hline Chnl-17 & T6 \\
\hline Chnl-18 & O1 \\
\hline Chnl-19 & O2 \\
\hline
\end{tabular}

\subsection{Data Collection}

In this study, three experimental paradigms were designed to prevent design biases. The tongue dynamics of 8 male and 2 female participants without any nervous system impairment can provide similar signal direction patterns of each tongue touchings. The participants were placed in front of the LCD monitor and guided not to move any part of the gross body except tongue motions during the task timing and trial procedure. However, eye movements were set free in the resting timing. Four trials for each experimental paradigm provided a total of 12 trials for each participant. Low intertrial variability and robust performances were observed between the trial results of each subject due to the complex muscles of the tongue and less effort $[1,5]$. Therefore preevaluated trials in terms of the classification results and the observed best trial of each subject were selected to compare the performances of DT and kNN methods. Then, complete processed results were provided using this data set of the trial. Statistical information of each participant was presented in Table 2.

Each recorded trial has $98 \mathrm{~s}$ and begins after the $10 \mathrm{~s}$ delay. Multiple touching contacts last $6 \mathrm{~s}$ duration for each right or left tongue movements in the oral cavity. Then, $5 \mathrm{~s}$ resting interval is inserted between the $6 \mathrm{~s}$ tongue motion durations and expected no tongue motion at this interval. The tongue is extended through forward in the intervals of the rest timing. Then, four right tongue movements and four left tongue movements were made by the subjects following to the experimental sequences shown in Fig.2. Approximately 10 multiple distinct contacts were implemented to the buccal walls during the 6 seconds. Distinct contacts are important to unveil the GKPs. Experimental paradigm sequences were arranged in different orders to reduce the memorizing effect for each subject. The equal direction number $(4$ right and 4 left) and timing process ( $6 \mathrm{~s}$ for task $5 \mathrm{~s}$ for resting) were made to be deterministic and boosting robust results in each experiment of the subjects. The subjects were guided by using powerpoint slide based experiments to reveal the patterns of GKP signals. Therefore inter-trial differences were compensated and reduced as possible as for each subject. The control of tongue movements in recorded signals were made by observation of amplitude and repetitional GKP signals $(<100 \mathrm{uV})$ which are much higher than the normal EEG signals $(10 \mathrm{uV}<\mathrm{EEG}<100 \mathrm{uV})$ or EOG artifacts.

Table 2. Selected experimental setup and trial number for each participant with statistical information

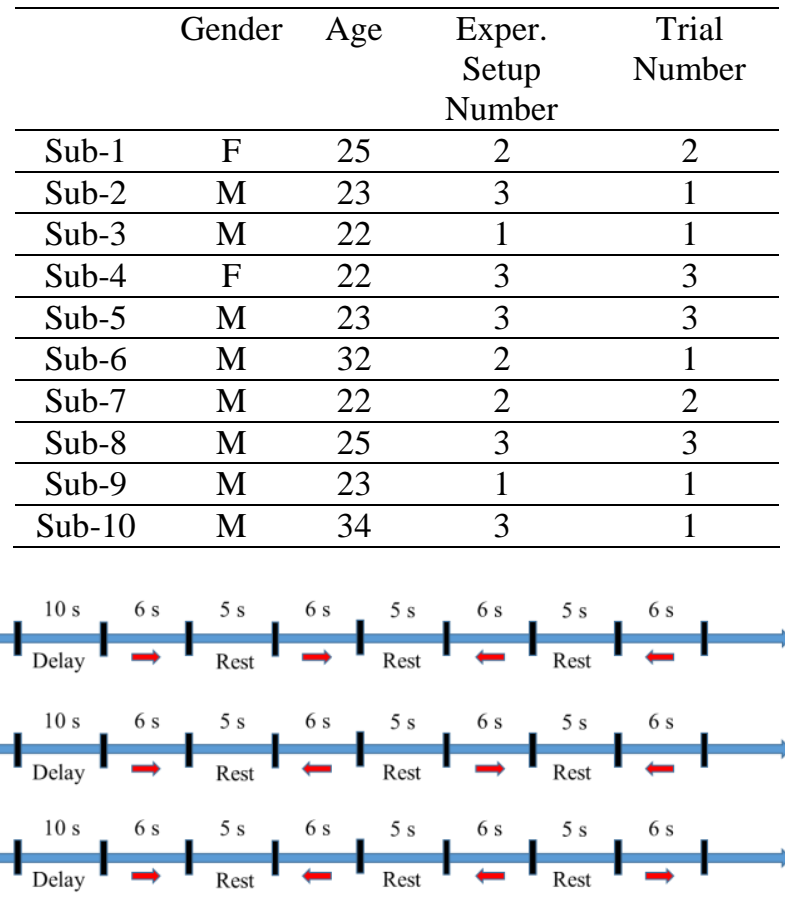

Fig.2. Sequences of the three experimental paradigms for tongue motions in the GKP-based TMI study

\subsection{Discrete Wavelet Transform}

Discrete Wavelet Transform (DWT) has revealed as a powerful tool to extract desired signals from non-stationary signals. DWT is also very suitable for multi-resolution analysis and consists of a series of operations for filtering and downsampling the input signal. Accomplishing scaling functions and wavelet functions are used with associated low and high pass filters to decompose high-frequency and lowfrequency components $[26,27]$. In this study, to achieve better classification results in feature extraction, the DWT technique was conducted for preprocessing and extraction of the delta and theta frequency bands of GKPs. In this application, the db10 of Wavelet type (Daubechies) at the 6th level was implemented to extract the delta and theta bands of GKPs for a sampling frequency of $1024 \mathrm{~Hz}$. 


$$
\operatorname{DWT}(a, b)=\frac{1}{\sqrt{a}} \sum_{n} x(n) \psi^{*}\left(\frac{n-b}{a}\right)
$$

In this expression, $a$ and $b$ are called scaling and translation parameters, respectively [28].

\subsection{Feature Extraction}

Feature extraction consists of calculation from the raw data signal to the informative and non-redundant feature vector. In this study, the Mean-Absolute Value (MAV-time domain) and Power Spectral Density (PSD-frequency domain) methods were applied. Feature extraction methods do not need transformation operation for time domain. However, frequency domain requires a transformation process from one domain to other [29]. The mathematical presentation of the mean-absolute value is defined in Eq.3.

$$
M A V=\frac{1}{N} \sum_{i=1}^{N}\left|x_{i}\right|
$$

where $X_{i}=1,2,3 \ldots N$ is a sequence of time, $N$ stands for the samples' length in the data array. Power signal changes of glossokinetic potential responses have different frequency ranges on the scalp that the PSD exhibits. PSD is a function describing the power distribution over a signal frequency. The mathematical formula of PSD is shown in Eqs.4-7:

$$
P x(f)=\frac{1}{N}\left|\sum_{n=0}^{N-1} x(n) e^{-j 2 \pi f n}\right|^{2}=\frac{1}{N}|X(f)|^{2}
$$

where $X(f)$ is the Fourier Transform of the data series of $x(n)$, $N$ means the samples' length. PSD of the signal is implemented by periodogram, and familiar kind of a periodogram is called Welch's method. In this method, data segments are separated and then overlapped as shown below:

$$
\begin{gathered}
x_{i}(n)=x(n+i D) \quad n=0,1, \ldots, M-1 \\
i=0,1, \ldots, L-1
\end{gathered}
$$

where $i$ shows the data segment, $n$ is the length of the segment. Moreover, $i D$ is specified as the first value for the ith sequence of $D=M$, and then the segments do not overlap. However, if the sequence occurs in $D=M / 2$, then $50 \%$ overlap is implemented over the data segments. Overall PSD is calculated applying the windowing process to each data segment. The modified periodogram is in Eq.6.

$$
\bar{P}_{X}^{(i)}(f)=\frac{1}{M U}\left|\sum_{n=0}^{N-1} x(n) w(n) e^{-j 2 \pi f n}\right|^{2}
$$

where $U$ is appointed as the normalization factor in the window functioning " $w(n)$ '":

$$
U=\frac{1}{M} \sum_{n=0}^{N-1} w^{2}(n)
$$

More knowledge for PSD and Welch's method can be seen in these references [30,31]. In our study, the data samples were divided into eight segments that are $50 \%$ overlapping and were processed with hamming windows. These eight segment and overlapping process were carried out by PSD for each segmentation before the frequency domain transformation.

The collected data constitutes a vector of length $(6 \times 8 \times 1024) \times 19$ for each participant. 6 stands for six seconds for multiple contacts in a trial, 8 is the number of total tongue movements (4 right-4 left), 1024 means the frequency of sampling and 19 states the channel numbers. It covers all EEG frequencies along feature extraction process, then 100 ms window was applied to generate the feature vector. 1 second data is $1024 / 100 \mathrm{~ms}=10$ parts (approx.), after that (6x8x10) equals the data length of 480 (approx.). However, some of the subjects start and end trials in difficulty at definite times during the experiments. Therefore, the data set had been cut off and defined it as a 400x19 dimension for each participant.

\subsection{Principal Component Analysis}

Principal Component Analysis (PCA) is a statistical method using orthogonal transform to calculate a set of observations of possibly related variables into a set of linearly uncorrelated variables, called principal components (PCs) [24,32]. PCA considers removing unnecessary data. Thus, it provides an easier calculation for machine learning (ML's) algorithms. Converting the high dimensional data $\left(X_{i}\right)$ is reduced into a lower dimensional data $\left(S_{t}\right)$ by PCA. The eigenvalues and eigenvectors are determined for the covariance matrix $(C)$ in this technique. The related equations are shown in (8-10).

$$
\begin{aligned}
C(X) & =\sum_{1}^{N} \frac{\left(X_{i} X^{T}\right)}{N} \\
u_{i} & =C u_{i}, \quad i=1,2,3 \ldots m
\end{aligned}
$$

where $\lambda_{i}$ stands for the eigenvalues and $u_{i}$ is the related eigenvector.

$$
S_{t}(i)=u_{t}^{T} X_{t}, \quad i=1,2,3 \ldots m
$$

where $S_{t}(i)$ describes the principal components of the data set $\left(X_{t}\right)$ [32,33]. In this research, 12-features were selected from the 19-features representing the highest variance values to form a $400 \times 12$ data set indicating all data between $97.9 \%$ $99.8 \%$ range. 


\subsection{Independent Component Analysis}

Independent Component Analysis (ICA) is a kind of statistical technique aiming to reveal concealed factors called independent components and to find linear projections of data that maximize mutual independence. Blind Source Separation (BSS) technique is based on ICA that intends to select the best EEG channels. Fewer EEG channels mean more portable and convenience interfaces and assistive technologies. ICA may be a useful tool to understand the functioning of brain mapping and glossokinetic potential responses during voluntary tongue movements $[34,35]$. The related equations of ICA are represented in 11-13.

$$
\begin{gathered}
x_{i}(t)=a_{i 1} s_{1}(t)+\cdots+a_{i n} s_{n}(t) \\
i=1,2, \ldots, n
\end{gathered}
$$

where $x_{i}(t)$ is the linear signal mixture belonging to $n$ differently and randomly varying coefficients, and $s_{n}(t)$ shows the hidden component [24]. ICA notation is in a matrix form:

$$
\begin{aligned}
& {\left[\begin{array}{c}
x_{1}(t) \\
\vdots \\
x_{n}(t)
\end{array}\right]=A\left[\begin{array}{c}
s_{1}(t) \\
\vdots \\
s_{n}(t)
\end{array}\right]} \\
& A=\left[a_{i 1}, a_{i 2}, \ldots, a_{i n}\right]
\end{aligned}
$$

Then,

$$
x=A s
$$

In terms of practical usage, a BCI system with fewer EEG channels which can be able to produce greater performance in term of the portability and convenience is preferable [34,35]. Based on the same basis, in this article, ICA and PCA were implemented to select and reduce the optimal channels respectively. For this reason, the channel numbers were reduced from the raw data set $(400 \times 19)$ to the data size $(400 \times 12)$ and compared the results with PCA $(400 \times 12)$. Hence, the success of the selection method (ICA) and projection technique (means PCA) was explored in terms of classification success of the GKP signals in a tonguemachine interface model.

\section{MACHINE LEARNING ALGORITHMS}

The relevant data set is processed by machine learning methods after preprocessing, feature extraction and feature selection operations. In this study, Decision Tree (DT) with Gini Index and k-Nearest Neighbors (kNN) algorithms were implemented to recognize the pattern of glossokinetic potential signals for extraction of 1-D related to touching of tongue movements on the buccal walls.

Accuracy (ACC), Sensitivity (SENS), Specificity (SPEC), and Information Transfer Rate (ITR) evaluation parameters were obtained to survey the performance of the GKP-based TMI. k-fold cross-validation is to improve the stability of the results. Then 10-fold was used in the calculations [36]. The equations represent for the correctness of the classification in Eqs.14-16.

$$
\begin{aligned}
& \operatorname{ACC}(T S)=\frac{\sum_{i=1}^{|T S|} \text { estimate }\left(n_{i}\right)}{|T S|}, \quad n_{i} \in T S \\
& \text { Estimate }(n)=\left\{\begin{array}{l}
1, \text { if estimate }(n)=c n \\
0, \text { otherwise }
\end{array}\right. \\
& \text { Class. ACC }=\frac{\sum_{i=1}^{|k|} \operatorname{accuracy}\left(T S_{i}\right)}{|k|}
\end{aligned}
$$

where $T S$ refers the test data set, then $n \in T S, c_{n}$ is the class of $n$. Furthermore, estimate( $n$ ) stands for the classification value for $n, k$ is the value for the $\mathrm{k}$-fold cross validation [24,36]. True negative (TN), true positive (TP), false negative (FN) and false positive (FP) are the primary parameters implemented for the specificity (SPEC) and sensitivity (SENS) in Receiver Operating Characteristic (ROC) analysis, represented in Eqs.17-18 [36].

$$
\begin{aligned}
& \text { Sensitivity }=\frac{T P}{T P+F N} \\
& \text { Specificity }=\frac{T N}{T N+F P}
\end{aligned}
$$

In EEG-based BCI systems, ITR is characterized a measuring parameter providing transmitted data information per trial or time. In this study, ITR was utilized to measure the system performance of GKP-based TMI. The equation of ITR is in Eq.19:

$$
B=\log _{2} N+P \log _{2} P+(1-P) \log _{2} \frac{(1-P)}{(N-1)}
$$

where $B$ describes the bits per trial, $N$ is different types of mental tasks. Then the accuracy is named as $P$. If the number of mental functions increases, the value of ITR increases in the range of (0-1) [37-39].

\subsection{Decision Trees}

Decision tree (DT) is known as a hierarchical data structure that is based on the divide-and-conquer strategy. This rulebased algorithm is called a nonparametric method, which creates a tree for a model in the training of data. Then this model is used to structure a set of simple rules. The new data set is carried out to be labeled for the classes in a sequencing recursive splits via implementing the internal decision nodes and terminal leaves [32,40]. In this article, the splitting criterion of the Decision Tree was selected as the Gini Index. The related equations are shown in Eqs. 20-22. 


$$
\begin{aligned}
& G i n i_{\text {left }}=1-\sum_{i=1}^{k}\left(\frac{L_{i}}{\left|T_{\text {left }}\right|}\right)^{2} \\
& G i n i_{\text {right }}=1-\sum_{i=1}^{k}\left(\frac{R_{i}}{\left|T_{\text {right }}\right|}\right)^{2}
\end{aligned}
$$

where $k$ is named as the total number of classes in the data set, $T$ states the number of samples in a node, $\left|T_{l e f t}\right|$ is called as the number of samples on the left branch, $\left|T_{\text {right }}\right|$ means the number of samples on the right branch, Ri is defined as the number of samples in the ith group at the right branch, and $L_{i}$ is the number of samples in the ith group at the left branch. The values of the relevant attributes are made in a group of pairs. The class values related to the left and right separations are provided in this way. Then Ginileft and Gini $i_{\text {right }}$ values are determined for each attribute in the left and right divisions as below:

$$
\operatorname{Gini}_{j}=\frac{1}{n}\left(\left|T_{\text {left }}\right| \cdot \text { Gini }_{\text {left }}+\left|T_{\text {right }}\right| \cdot \text { Gini }_{\text {right }}\right)
$$

The calculated minimum Gini values for the relevant $j$ attribute is chosen as a division attribute [41]. The procedure is repeated until the leaf node is reached for the final decision.

\section{2. k-Nearest Neighbors}

This Euclidean-based algorithm assigns an input data between the k-Nearest Neighbors $(\mathrm{kNN})$ of having most examples in the training set. All neighbors receive an equal vote and class having a maximum number of voters among its neighbors is selected. The degree of smoothing parameter is controlled by $k$ in the neighborhood of $\mathrm{kNN}$ [32]. Thus, $k$ must be smaller than $N$ (length of data). Mathematical approaches for Euclidean distance between $a$ and $b$ for each sample:

$$
d_{1}(x) \leq d_{2}(x) \leq \cdots \leq d_{N}(x)
$$

Distances are located in ascending order by the nearest distance $d_{1}(x)$ and the next nearest distance $d_{2}(x) . x^{t}$ is described as data points, and $t$ is the index of neighborhoods of samples:

$$
d_{1}(x)=\min _{t}\left|x-x^{t}\right|
$$

In order to obtain the best results in this study, step k has been searched between 3 and 25 [29,42].

\section{RESULTS}

GKPs were used to advance a TMI for 1-D control. MAV and PSD were employed in DT with Gini Index and kNN algorithms. In this work, the raw data set size was calculated as $400 \times 19$ dimension for each participant. The results in the article were processed by MLs from the data sets:

- The raw data set $(400 x 19)$

- $\quad$ The reduced data set processed by PCA $(400 \times 12)$

- $\quad$ The reduced data set processed by ICA (400x12)

- The Extracted frontal lobe data set (7 channels) (400x7)

- The Extracted frontal and temporal lobes data set (11 channels) (400x11)

All the results on the paper are shown in the decimal base and percentage expression (\%), excluding ITR results. The best and worst participants were determined by observing the raw data set results to

\begin{tabular}{|c|c|c|c|c|c|c|c|c|c|c|c|c|}
\hline Method & . & Sb1 & Sb2 & Sb3 & Sb4 & Sb5 & Sb6 & Sb7 & Sb8 & Sb9 & Sb10 & Aver. \\
\hline \multirow{4}{*}{ 它 } & Acc & 71.17 & 94.27 & 70.27 & 71.09 & 88.06 & 93.21 & 83.01 & 75.66 & 77.70 & 91.04 & 81.55 \\
\hline & Sen & 76.17 & 94.50 & 74.15 & 75.29 & 91.23 & 93.28 & 84.48 & 77.65 & 79.62 & 91.78 & 83.81 \\
\hline & Spe & 64.24 & 93.95 & 65.26 & 64.82 & 83.41 & 93.32 & 81.48 & 73.12 & 75.41 & 90.08 & 78.51 \\
\hline & ITR & 0.133 & 0.684 & 0.122 & 0.132 & 0.472 & 0.642 & 0.342 & 0.199 & 0.234 & 0.565 & 0.353 \\
\hline \multirow{4}{*}{ 占氞 } & Acc & 75.53 & 93.03 & 71.14 & 70.05 & 86.49 & 94.16 & 83.54 & 76.22 & 77.00 & 91.29 & 81.84 \\
\hline & Sen & 81.88 & 91.50 & 75.95 & 74.87 & 90.78 & 95.40 & 83.55 & 78.54 & 80.09 & 93.93 & 84.65 \\
\hline & Spe & 66.97 & 94.57 & 65.18 & 63.58 & 80.11 & 92.48 & 83.35 & 73.51 & 72.71 & 87.88 & 78.03 \\
\hline & ITR & 0.197 & 0.635 & 0.133 & 0.119 & 0.429 & 0.679 & 0.355 & 0.209 & 0.222 & 0.573 & 0.355 \\
\hline \multirow{4}{*}{ Z } & Acc & 70.27 & 96.02 & 67.20 & 68.32 & 87.01 & 94.30 & 83.02 & 73.07 & 80.98 & 92.50 & 81.27 \\
\hline & Sen & 85.24 & 95.50 & 90.04 & 74.37 & 89.86 & 96.21 & 80.05 & 72.45 & 78.79 & 96.03 & 85.85 \\
\hline & Spe & 50.44 & 96.55 & 37.78 & 60.19 & 83.22 & 91.35 & 86.27 & 73.76 & 84.05 & 87.58 & 75.12 \\
\hline & ITR & 0.122 & 0.759 & 0.087 & 0.099 & 0.443 & 0.685 & 0.343 & 0.160 & 0.298 & 0.616 & 0.361 \\
\hline \multirow{4}{*}{ 艺 } & Acc & 70.66 & 95.26 & 68.16 & 69.16 & 86.15 & 94.17 & 81.02 & 72.54 & 77.09 & 91.02 & 80.52 \\
\hline & Sen & 82.64 & 95.44 & 89.85 & 77.76 & 89.39 & 97.05 & 81.29 & 73.22 & 75.33 & 95.22 & 85.72 \\
\hline & Spe & 54.99 & 95.04 & 40.24 & 57.59 & 81.38 & 90.04 & 80.56 & 71.82 & 79.48 & 85.32 & 73.64 \\
\hline & ITR & 0.127 & 0.725 & 0.097 & 0.109 & 0.420 & 0.679 & 0.299 & 0.152 & 0.224 & 0.564 & 0.340 \\
\hline
\end{tabular}
compare easily. Then, the statements for outcomes were explained related to the participants of best and worst in the article.

Table 3. Machine learning performances for the raw data set $(400 \times 19)$ 
Table 4. Machine learning performances for the reduced data set by PCA $(400 \times 12)$

\begin{tabular}{|c|c|c|c|c|c|c|c|c|c|c|c|c|}
\hline Method & .. & Sb1 & Sb2 & Sb3 & Sb4 & Sb5 & Sb6 & Sb7 & Sb8 & Sb9 & Sb10 & Aver. \\
\hline \multirow{4}{*}{ 占㐫 } & Acc & 73.17 & 95.01 & 71.21 & 69.47 & 88.02 & 91.03 & 82.08 & 75.41 & 78.06 & 90.37 & 81.38 \\
\hline & Sen & 77.96 & 94.00 & 77.79 & 77.43 & 89.11 & 91.11 & 82.60 & 78.04 & 79.66 & 93.04 & 84.07 \\
\hline & Spe & 67.17 & 96.02 & 62.17 & 57.96 & 85.60 & 90.81 & 81.66 & 72.31 & 75.68 & 86.14 & 77.55 \\
\hline & ITR & 0.161 & 0.714 & 0.134 & 0.112 & 0.471 & 0.565 & 0.322 & 0.195 & 0.241 & 0.543 & 0.346 \\
\hline \multirow{4}{*}{ 约氙 } & Acc & 73.01 & 92.27 & 71.21 & 68.28 & 84.08 & 93.30 & 80.49 & 74.11 & 80.47 & 91.20 & 80.84 \\
\hline & Sen & 76.56 & 89.50 & 77.31 & 70.54 & 88.70 & 94.93 & 81.30 & 76.36 & 81.01 & 92.23 & 82.84 \\
\hline & Spe & 68.63 & 94.93 & 63.53 & 65.21 & 77.14 & 91.01 & 79.51 & 71.35 & 79.58 & 89.62 & 78.05 \\
\hline & ITR & 0.159 & 0.607 & 0.134 & 0.099 & 0.368 & 0.646 & 0.288 & 0.175 & 0.288 & 0.570 & 0.333 \\
\hline \multirow{4}{*}{ Z } & Acc & 73.35 & 95.01 & 72.93 & 70.18 & 87.07 & 95.82 & 81.01 & 73.53 & 79.74 & 93.07 & 82.17 \\
\hline & Sen & 82.83 & 93.17 & 87.76 & 75.30 & 88.92 & 97.90 & 79.56 & 71.98 & 78.77 & 95.76 & 85.19 \\
\hline & Spe & 60.53 & 96.85 & 53.92 & 62.26 & 83.83 & 93.12 & 82.26 & 75.76 & 81.43 & 89.43 & 77.94 \\
\hline & ITR & 0.164 & 0.714 & 0.157 & 0.121 & 0.444 & 0.749 & 0.299 & 0.166 & 0.273 & 0.637 & 0.372 \\
\hline \multirow{4}{*}{ 荤金 } & Acc & 74.02 & 90.70 & 71.32 & 67.66 & 84.35 & 93.05 & 78.93 & 74.56 & 74.36 & 88.04 & 79.70 \\
\hline & Sen & 86.20 & 87.50 & 80.85 & 73.10 & 84.83 & 95.36 & 79.64 & 77.25 & 74.87 & 95.22 & 83.48 \\
\hline & Spe & 57.97 & 93.85 & 58.22 & 60.59 & 83.01 & 89.87 & 78.23 & 71.18 & 73.85 & 78.16 & 74.49 \\
\hline & ITR & 0.174 & 0.553 & 0.135 & 0.092 & 0.374 & 0.636 & 0.257 & 0.182 & 0.179 & 0.472 & 0.305 \\
\hline
\end{tabular}

* Accuracy (Acc),Specificity (Spe) and Sensitivity (Sen)

As seen in Table 3, the highest and lowest classification accuracies of glossokinetic potential-based right-left tongue movements were obtained as $96.02 \%$ and $67.20 \%$ by $\mathrm{kNN}+\mathrm{MAV}$ methods, respectively. Then, the best and worst subjects were defined according to the raw data set results as Sub-2 (96.02\%) and Sub-3 (67.20\%) respectively. Moreover, for the Sub-3, the greatest outcome was provided by DT+PSD $(71.14 \%)$. Thus, the difference value between the results of the highest accuracies for the best and worst subjects is 24.88. Moreover, DT+PSD have outperformed for the highest average accuracy of $81.84 \%$. Furthermore, $\mathrm{DT}+\mathrm{MAV}, \mathrm{kNN}+\mathrm{MAV}$, and $\mathrm{kNN}+\mathrm{PSD}$ obtained very close outcomes in terms of average classification accuracies around $80-81 \%$. In Fig.3, the best and worst subject performances are shown based on the raw data set outcomes.
Reduced data set results implementing PCA technique can be seen in Table 4. The best subject performed reaching up to the $95.01 \%$ classification accuracy by $\mathrm{kNN}+\mathrm{MAV}$. Then the worst participant obtained accuracy of $72.93 \%$ with the same method (kNN+MAV). The decrease value for the best participant compared to the raw data set result (Table 3) was achieved as $1.05 \%$. However, $2.51 \%$ increase in accuracy was provided by reduced data set after the PCA technique. Additionally, the average classification accuracy was improved and obtained by kNN+MAV (82.17\%) compared to the raw data set result $(81.84 \%)$. Easier computation of classifiers may cause this increment due to the lower dimension of data processed by PCA. Hence the spatial pattern of glossokinetic potential signals can be recognized better after the redundant data by PCA.

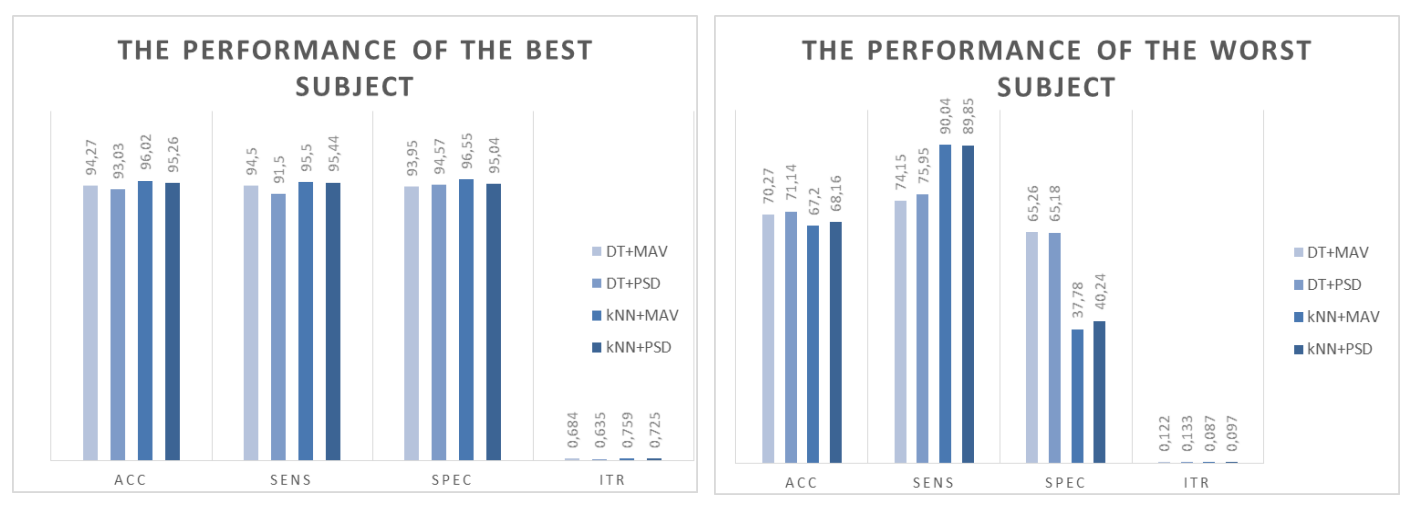

Fig.3. a. Classification performances of the best subject (left) b. Classification performances of the worst subject (right) 
Table 5. Machine learning performances for the reduced data set by ICA $(400 \times 12)$

\begin{tabular}{|c|c|c|c|c|c|c|c|c|c|c|c|c|}
\hline Tethod & & Sb1 & Sb2 & Sb3 & Sb4 & Sb5 & Sb6 & Sb7 & Sb8 & Sb9 & Sb10 & Aver. \\
\hline \multirow{4}{*}{$\bar{\theta}$} & Acc & 72.13 & 88.13 & 71.65 & 71.20 & 82.04 & 87.22 & 79.00 & 75.47 & 77.54 & 83.19 & 78.76 \\
\hline & Sen & 74.43 & 89.50 & 76.50 & 74.89 & 85.82 & 89.00 & 79.68 & 79.55 & 81.34 & 85.27 & 81.60 \\
\hline & Spe & 69.27 & 86.77 & 65.32 & 66.56 & 76.52 & 84.13 & 78.35 & 70.70 & 72.17 & 80.46 & 75.03 \\
\hline & ITR & 0.146 & 0.474 & 0.140 & 0.134 & 0.321 & 0.449 & 0.258 & 0.196 & 0.231 & 0.347 & 0.270 \\
\hline \multirow{4}{*}{ 它食 } & Acc & 71.21 & 84.24 & 70.01 & 72.41 & 80.25 & 86.34 & 76.75 & 76.28 & 78.25 & 05 & 77.88 \\
\hline & Sen & 74.03 & 85.00 & 72.87 & 77.05 & 84.98 & 87.34 & 77.38 & 79.03 & 80.09 & 86.11 & 80.39 \\
\hline & Spe & 67.45 & 83.57 & 66.75 & 65.38 & 74.18 & 84.85 & 76.04 & 72.24 & 75.82 & 78.56 & 74.48 \\
\hline & ITR & 0.134 & 0.372 & 0.119 & 0.150 & 0.283 & 0.425 & 0.218 & 0.210 & 0.244 & 0.343 & 0.250 \\
\hline \multirow{4}{*}{ 否离 } & Acc & 72.33 & 91.51 & 73.32 & 72.58 & 85.21 & 91.33 & 78.52 & 76.22 & 80.23 & 89.26 & 81.05 \\
\hline & Sen & 87.66 & 93.98 & 80.91 & 73.12 & 87.43 & 95.78 & 70.31 & 75.89 & 74.95 & 96.93 & 83.70 \\
\hline & Spe & 52.27 & 89.06 & 64.49 & 71.84 & 81.93 & 84.97 & 87.97 & 76.64 & 87.63 & 78.81 & 77.56 \\
\hline & ITR & 0.149 & 0.581 & 0.163 & 0.153 & 0.395 & 0.575 & 0.249 & 0.209 & 0.283 & 0.508 & 0.326 \\
\hline \multirow{4}{*}{ 药食 } & Acc & 72.09 & 87.35 & 69.04 & 69.24 & 83.04 & 89.28 & 75.48 & 75.37 & 78.96 & 87.19 & 78.70 \\
\hline & Sen & 87.40 & 81.37 & 85.07 & 76.94 & 83.32 & 97.13 & 76.37 & 79.54 & 79.20 & 93.90 & 84.02 \\
\hline & Spe & 51.74 & 93.34 & 48.43 & 59.13 & 83.11 & 77.41 & 74.58 & 70.16 & 78.16 & 77.91 & 71.40 \\
\hline & ITR & 0.146 & 0.452 & 0.107 & 0.110 & 0.343 & 0.509 & 0.196 & 0.195 & 0.258 & 0.448 & 0.276 \\
\hline
\end{tabular}

Table 6. Machine learning performances for the frontal lobe signal data set $(400 \times 7)$

\begin{tabular}{|c|c|c|c|c|c|c|c|c|c|c|c|c|}
\hline Method & .. & Sb1 & Sb2 & Sb3 & Sb4 & Sb5 & Sb6 & Sb7 & Sb8 & Sb9 & Sb10 & Aver. \\
\hline \multirow{4}{*}{ 鸟 } & Acc & 69.01 & 95.07 & 68.39 & 69.54 & 80.54 & 92.59 & 80.11 & 62.09 & 72.14 & 91.18 & 78.07 \\
\hline & Sen & 73.46 & 95.00 & 76.94 & 72.30 & 86.58 & 93.70 & 83.51 & 66.52 & 78.37 & 92.72 & 81.91 \\
\hline & Spe & 62.19 & 95.21 & 57.57 & 66.26 & 71.48 & 91.02 & 76.32 & 56.48 & 63.70 & 88.84 & 72.91 \\
\hline & ITR & 0.107 & 0.717 & 0.100 & 0.113 & 0.289 & 0.619 & 0.280 & 0.043 & 0.146 & 0.570 & 0.298 \\
\hline \multirow{4}{*}{$\widehat{\theta}$} & Acc & 70.01 & 95.03 & 69.17 & 71.13 & 80.22 & 94.16 & 82.37 & 64.02 & 75.48 & 91.02 & 79.26 \\
\hline & Sen & 75.73 & 95.50 & 75.63 & 76.61 & 84.93 & 96.63 & 82.58 & 69.66 & 78.77 & 89.60 & 82.56 \\
\hline & Spe & 62.40 & 94.46 & 61.29 & 63.65 & 73.50 & 90.81 & 82.06 & 57.04 & 71.24 & 92.81 & 74.93 \\
\hline & ITR & 0.119 & 0.715 & 0.109 & 0.133 & 0.283 & 0.679 & 0.328 & 0.058 & 0.196 & 0.564 & 0.318 \\
\hline \multirow{4}{*}{ 宓妾 } & Acc & 69.23 & 96.77 & 68.35 & 67.12 & 83.05 & 94.05 & 82.04 & 61.44 & 77.70 & 91.76 & 79.15 \\
\hline & Sen & 82.14 & 97.25 & 88.36 & 73.17 & 84.64 & 94.47 & 79.27 & 75.72 & 77.48 & 93.06 & 84.56 \\
\hline & Spe & 52.45 & 96.36 & 42.91 & 59.23 & 80.99 & 93.13 & 85.46 & 42.89 & 77.85 & 90.39 & 72.17 \\
\hline & ITR & 0.110 & 0.794 & 0.099 & 0.086 & 0.344 & 0.675 & 0.321 & 0.038 & 0.234 & 0.589 & 0.329 \\
\hline \multirow{4}{*}{ 蒙食 } & Acc & 70.28 & 96.33 & 68.11 & 66.02 & 82.05 & 94.25 & 82.51 & 60.02 & 74.43 & 89.85 & 78.39 \\
\hline & Sen & 83.13 & 95.94 & 86.38 & 75.15 & 86.13 & 94.51 & 81.69 & 70.80 & 73.61 & 91.74 & 83.91 \\
\hline & Spe & 54.08 & 96.81 & 44.37 & 53.28 & 75.85 & 93.65 & 83.42 & 46.46 & 76.07 & 87.57 & 71.16 \\
\hline & ITR & 0.122 & 0.773 & 0.097 & 0.075 & 0.321 & 0.683 & 0.331 & 0.029 & 0.180 & 0.526 & 0.314 \\
\hline
\end{tabular}

In Table 5, ICA has a great average and individual performances in reduced data set by twelve features. The best and worst participants have obtained the highest accuracy of $91.51 \%$ and $73.32 \%$ with $\mathrm{kNN}+\mathrm{MAV}$ respectively. Then the average performances were again shown in the kNN+MAV method reaching up to the $81.05 \%$. Furthermore, ICA results are fairly close to the results of the PCA technique regarding the average outcomes.

Frontal and temporal lobes are responsible for planning, attention, conscious motor movement and language-speech recognition [37]. Then voluntary gross tongue movements might require focus and planning efforts in distinct and regular motions. Then these might lead EEG potentials, as well as GKPs during the experimental work. On the other hand, in the mechanism of generating glossokinetic potential responses, forward movements of the tongue may cause high-density GKP signals over the frontal and mastoid region $[43,44]$. Therefore in our research, the investigation of the classification effect of the frontal and temporal lobes was observed during voluntary tongue movements in Tables 6 and 7. Therefore, $400 \times 7$ and $400 \times 11$ data sets were extracted from the raw data set $(400 \times 19)$. T3, T4, T5, and T6 electrodes were implemented for eleven channels (Frontal + Temporal Lobe) as well as these seven electrodes for the seven channels (Fp1, Fp2, F7, F8, Fz, F3, and F4) (Frontal Lobe), as seen in Table 1.

In Table 6, the best and worst participant provided the highest accuracy of $96.77 \%$ (kNN+MAV) and $69.17 \%$ (DT+PSD). Then the greatest average accuracy of $79.26 \%$ was obtained by DT+PSD. 
Comparing to the raw data set results in Table 3 shows that the best subject's results have reached to $96.77 \%$, and the worst participant has $69.19 \%$ classification accuracy. Frontal and temporal lobe signals were processed together and evaluated to show the effects of both region in Table 7. The greatest outcomes of best and worst participants were obtained by $\mathrm{kNN}+\mathrm{MAV}(96.66 \%)$ and DT+PSD $(71.05 \%)$ respectively. Furthermore, the highest average accuracy $(82.49 \%)$ was obtained by kNN+MAV. Therefore the changes compared to raw data set results are as follows: for the best subject $(0.66 \%$ increment $)$, for the worst subject $(0.12 \%$ decrement $)$ and the average success $(0.79 \%$ increment) were achieved.

Table 7. Machine learning performances for the frontal+temporal lobe signals data set $(400 \times 11)$

\begin{tabular}{|c|c|c|c|c|c|c|c|c|c|c|c|c|}
\hline Method & & Sb1 & Sb2 & Sb3 & Sb4 & Sb5 & Sb6 & Sb7 & Sb8 & Sb9 & Sb10 & Aver. \\
\hline \multirow{4}{*}{ 宫离 } & Acc & 72.78 & 95.22 & 70.26 & 70.09 & 86.30 & 95.03 & 83.22 & 76.17 & 77.48 & 91.71 & 81.83 \\
\hline & Sen & 74.96 & 96.00 & 75.06 & 72.64 & 89.11 & 96.63 & 81.05 & 81.70 & 81.79 & 92.23 & 84.12 \\
\hline & Spe & 69.51 & 94.59 & 63.73 & 66.95 & 81.80 & 92.76 & 85.67 & 68.80 & 71.53 & 91.27 & 78.66 \\
\hline & ITR & 0.155 & 0.723 & 0.122 & 0.120 & 0.424 & 0.715 & 0.347 & 0.208 & 0.230 & 0.588 & 0.363 \\
\hline \multirow{4}{*}{ 氞 } & Acc & 74.42 & 93.29 & 71.05 & 68.57 & 85.31 & 95. & 83.05 & 75.25 & 76.02 & 91.01 & 81.30 \\
\hline & Sen & 79.70 & 91.50 & 78.68 & 73.61 & 90.34 & 95.36 & 80.80 & 79.39 & 78.35 & 91.30 & 83.90 \\
\hline & Spe & 67.14 & 95.13 & 60.63 & 61.69 & 77.48 & 93.94 & 85.49 & 70.16 & 72.86 & 90.65 & 77.52 \\
\hline & ITR & 0.180 & 0.645 & 0.132 & 0.102 & 0.398 & 0.714 & 0.343 & 0.193 & 0.205 & 0.564 & 0.348 \\
\hline \multirow{4}{*}{ 否交 } & Acc & 73.25 & 96.66 & 70.44 & 68.05 & 86.18 & 94.52 & 83.04 & 76.28 & 81.77 & 94.73 & 82.49 \\
\hline & Sen & 85.33 & 96.67 & 81.40 & 74.91 & 86.70 & 97.46 & 81.33 & 78.14 & 82.25 & 94.78 & 85.90 \\
\hline & Spe & 57.48 & 96.56 & 56.19 & 58.72 & 85.62 & 89.99 & 85.09 & 73.89 & 80.74 & 94.72 & 77.90 \\
\hline & ITR & 0.162 & 0.789 & 0.124 & 0.096 & 0.420 & 0.694 & 0.343 & 0.210 & 0.315 & 0.702 & 0.386 \\
\hline \multirow{4}{*}{ 茫食 } & Acc & 73.07 & 96.30 & 69.30 & 66.40 & 86.29 & 94.32 & 82.11 & 74.40 & 80.70 & 91.13 & 81.40 \\
\hline & Sen & 84.36 & 95.82 & 88.70 & 73.86 & 87.22 & 96.20 & 81.17 & 75.08 & 80.94 & 92.63 & 85.60 \\
\hline & Spe & 58.38 & 96.78 & 44.37 & 56.54 & 85.37 & 91.80 & 82.98 & 73.65 & 80.43 & 88.92 & 75.92 \\
\hline & ITR & 0.160 & 0.772 & 0.110 & 0.079 & 0.424 & 0.685 & 0.322 & 0.179 & 0.292 & 0.568 & 0.359 \\
\hline
\end{tabular}

a)

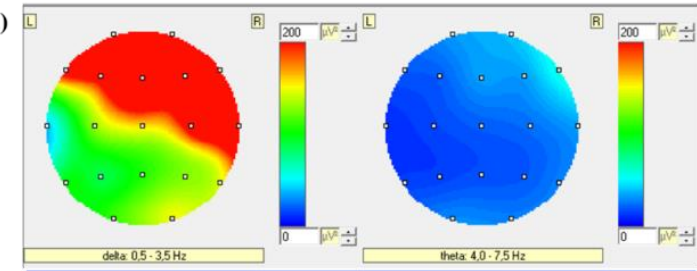

b)

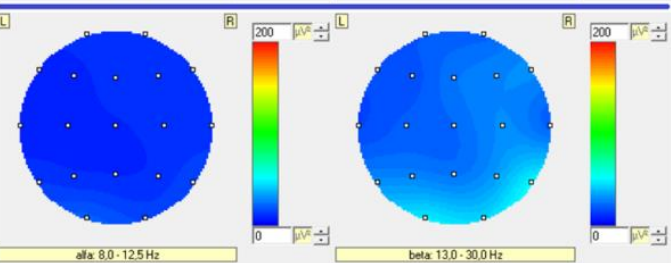

Fig.4. Brain mappings of Subject-2 (best participant) related the tongue movements towards touching the right buccal wall (a- the intensity of delta and theta bands $\mathbf{b}-$ the intensity alpha and beta bands)

Concentrated participants have highly signal variations on delta and theta bands [5]. As presented in Figs.4 and 5, high power alterations of the best subject can be observed over the frontal and temporal lobes and partly pre-motor and motor cortex on delta bands. This significant finding was compatible with the classification achievement shown in Tables 6 and 7. Furthermore, in theta and alpha frequency bands negligible power assessments were obtained to distinguish the certain GKP responses. However, insufficient power signal variations were observed in the beta bands of the occipital lobe at the contralateral side of the brain. This may depend on the visual stimulus in front of the LCD monitor or may be occurred by tongue touching the articulators in the oral cavity, such as palate [24,43-44].

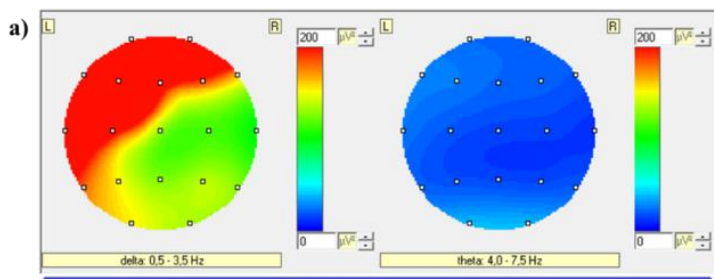

b)

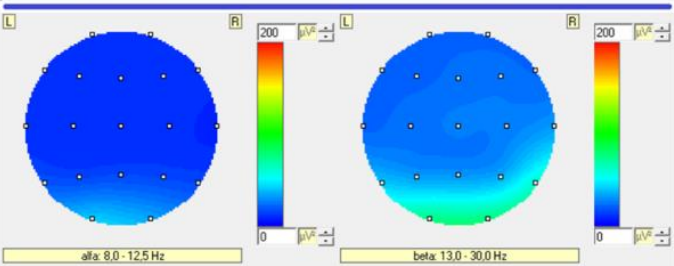

Fig.5. Brain mappings of Subject-2 (best participant) related the tongue movements towards touching the left buccal wall (a- the intensity of delta and theta bands $\mathbf{b}$ - the intensity of alpha and beta bands) 


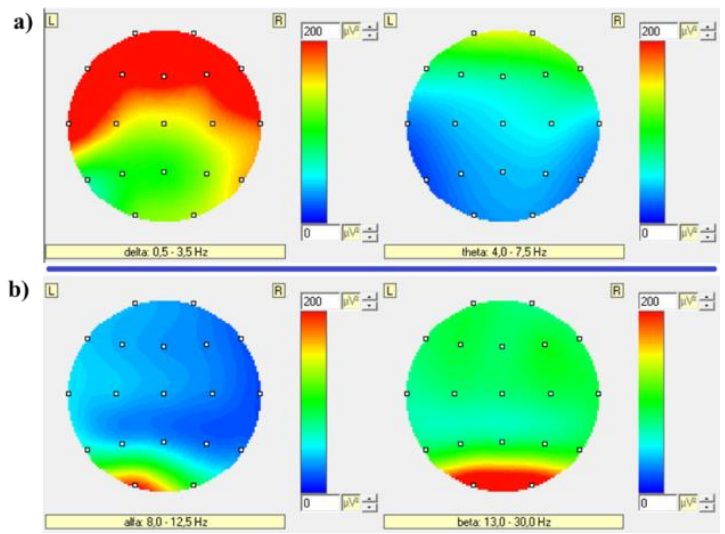

Fig.6. Brain mappings of Subject-3 (worst participant) related the tongue movements towards touching the right buccal wall (a- the intensity of delta and theta bands b- the intensity of alpha and beta bands)

The topography results of the best participant show that the frontal lobe has high-intensity power signal changes, but does not extend correctly and smoothly in the temporal lobes of the delta and theta frequencies including the T3, T4, T5, and T6 electrode locations introduced in Figs. 6 and 7.

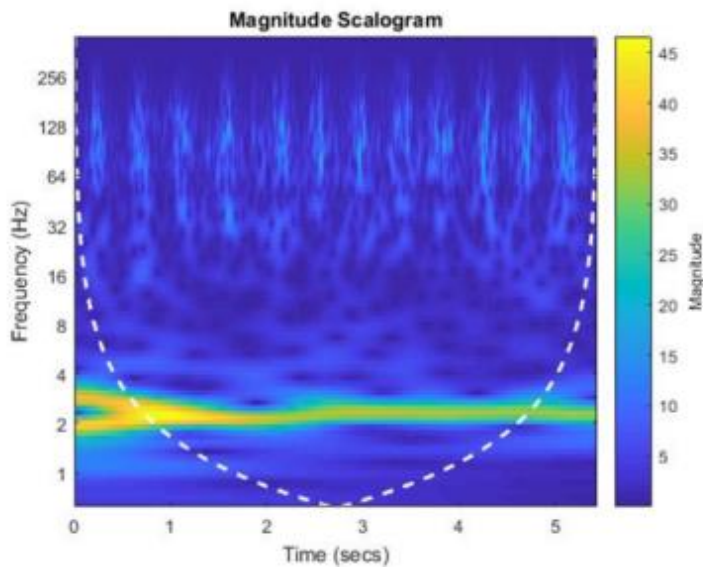

On the other hand, beta frequency bands of the worst subject have acceptable values compared to the best subject. The reason for this fact may be occurred due to the inadequate target-oriented motivation and disturbing perception $[37,45]$. Moreover, time-frequency analysis and scatter plot of classification for the best subject were presented in Fig.8.

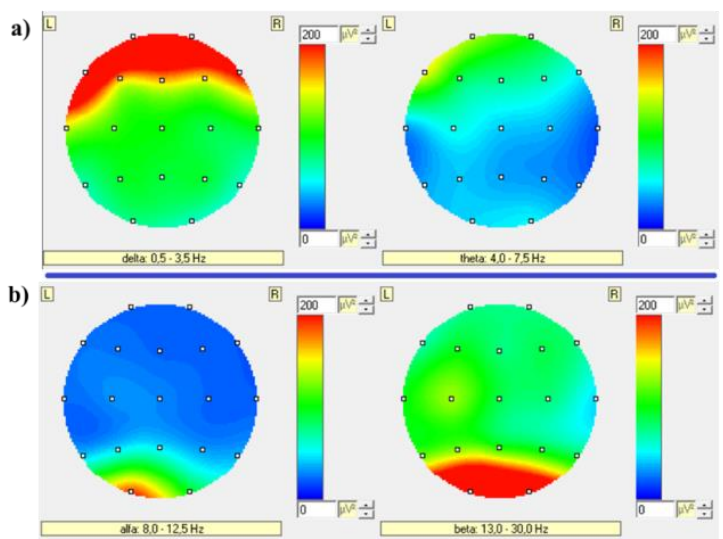

Fig.7. Brain mappings of Subject-3 (worst participant) related the tongue movements towards touching the left buccal wall (a- the intensity of delta and theta bands b- the intensity of alpha and beta bands)

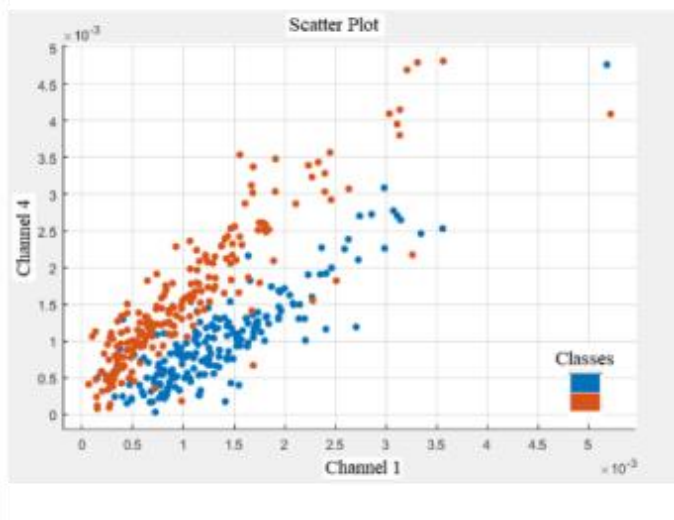

Fig.8. a- Time-frequency analysis (usage of Continuous Wavelet Transform) while touching the right buccal wall of the best participant during $6 \mathrm{~s}$ (left) b- Scatter plot presentation of the best subject (Channel-1 vs. Channel-4) (right)

Table 8. Computation times of the Decision Tree and kNearest Neighbor algorithms

\begin{tabular}{ccccc}
\hline Methods & $\begin{array}{c}\text { DT+ } \\
\text { MAV }\end{array}$ & DT+PSD & kNN+MAV & kNN+PSD \\
\hline $\begin{array}{c}\text { F.E.T.+ } \\
\text { C.T. (s) }\end{array}$ & 0.0014 & 0.9389 & 0.0021 & 0.9524 \\
\hline
\end{tabular}

F.E.T.(Feature Extraction Time), C.T. (Classfying Time)

Computation time is an important parameter to show the real-time application of the glossokinetic potential-based tongue machine interface $[1,4]$. Related to the Table 8 , DT+MAV has the least computation time with $0.0014 \mathrm{~s}$ among the other methods. Then the longest computation time was obtained by $\mathrm{kNN}+\mathrm{PSD}$ as 0.9524 s. Hence meanabsolute value is more appropriate than the power-spectral methods due to the not requiring transformation from the time domain to the frequency domain for real-time usage of the GKP-based TMI study [24,29]. The results represented in Table 8 were acquired in 1-fold cross-validation for average test samples of the raw data set of the best participant and computed using Matlab (License No: 834260). The computer hardware and software are those : Intel Core i5$7200 \mathrm{U}$ CPU $(2.50 \mathrm{GHz}), 64$ bit, 8 GB Ram and Windows 10 operating system.

\section{DISCUSSION}

The aim of this research is to investigate DT and kNN machine learning algorithms' performances in advancing a natural, attractive and reliable system using GKPs. Hence, these GKP responses are generated by distinct and simple tongue touches on the buccal walls. In this work, the results obtained show that the kNN+MAV and DT+PSD methods generally provide the highest classification scores for the 
individual and average success among other combination methods. In addition, the PSD feature extraction method takes prolonged time than the MAV in terms of the computation time. However, feature extraction performances depend on the data sets because of the smoothness or outliers. Machine learning algorithms, Decision Trees, and k-Nearest Neighbors have some advantages over the other classifiers. The Decision Trees are rule-based algorithms and easily interpreted and comprehended by experts. Then, kNN can give good results if appropriate feature extraction methods are applied [40]. Therefore, both of the classifiers performances have almost the same in raw and preprocessed data sets. Hence, the comparison of the Decision Trees and k-Nearest Neighbors in recognizing the spatial pattern of glossokinetic potential signals were explored as the first attempt among the machine learning algorithms in a tongue machine interface research.

Modern brain-machine interface (BMI) design requires multiple science disciplines. These efforts associate signal processing, information systems, control theory and machine learning [46-48]. The significant investigation of the research is about the contribution and assessment of frontal (7-channels) and frontal+temporal (11-channels) region signals due to the high exposure of GKPs regarding the success of the GKP-based TMI with fewer electrodes. Then the accuracy of the frontal + temporal region signals is very close to, or even better than the raw data set results, represented in Tables 7 and 3, respectively. This clarity may have been originated from the intense exposure of the glossokinetic potential variations over the frontal+temporal regions of the scalp. Then, this evidence was also promoted by brain mappings, as shown in Figs. 4 and 5. This finding might cause a significant increase in degrees of freedom challenge and reliability in tongue-machine interfaces due to the fewer channels [10-11, 35]. Therefore, easy-to-use, wearable, and portable systems can be designed on these promising findings for disabled persons [49]. Moreover, corticomuscular coupling analysis explains the relation of brain regions and ongoing muscular activities (EMG). Furthermore, coherence of cortex potentials and GKPs in delta and theta bands during the tongue-muscle motor functions were investigated for the first time in a TMI as our best knowledge [50].

The symmetrical situation was observed while occurring of GKPs on the scalp. As mentioned before, GKP signals were arisen unsymmetrically in the brain mappings due to the negatively charged tongue tip creating potentially increased and decreased variations on the non-contact and contact surfaces, respectively [3-5]. However, the same researchers reported that GKPs create dissimilar patterns in brain maps when tongue touches to the other articulators, teeth or palate. Moreover, pronouncing the retroflex consonants lead to a strong potential rising over the frontal region during the tongue bending in language and phonetic research [44]. It may be that in our study, an antisymmetric event may have been suppressed due to strong and distinct touchings while the tongue contacts the buccal walls during experimental tasks. Hence, our results may have revealed symmetrical formation on the brain mappings, represented in Figs. 4 and 5. According to the same article, these are different including the electrode placements, reference point, and experimental procedure. In their study, the tongue was moved in a continuous motion on the right-front-left path to touch the buccal walls [3,4]. However, in our research, multiple distinct contacts were carried out in the $6 \mathrm{~s}$ task. Thus, all these differences may form symmetrical results on brain maps in our study.

The performances of the best and worst participants were observed, and the comments were made to reveal the distinguishing features. The outcomes of the best participant show that distinct, speedy and regular tongue movements in high motivation state provide the significant achievements in GKP-based TMI. However, the success of the worst participant was acceptable due to the less concentration and not correctly doing instructed tasks. Motivation and cognitive effort have been identified as key parameters for the superior performance in BCIs [45,51].

The investigation of independent and principal component analysis on glossokinetic potential responses in our study is the first and considerable attempt. While ICA is better suited for non-Gaussian functions, PCA has great performance for smooth data of EEG signals. This truth may be the same for glossokinetic potential responses. Therefore, according to the Tables 4 and 5, PCA and ICA results are so close to each other. However, PCA is relatively better than ICA, especially for the best participant. Because of this reason may be that the data set of the best subject has more smooth and lower spatial overlapping of cortical activity [52-54]. Moreover, the extraction of the delta and theta bands with DWT is an alternative solution in the preprocessing stage for removing noise on GKPs. Also, DWT may produce better performances in classification success $[55,56]$.

The notion of inter-trial and inter-subject instability is an important issue for the performance of BCI research and a solution is named as transfer learning technique [46]. The same deduction has occurred but not as much as BCI research. Mostly low variations have been observed for each participant at the trial-to-trial performances. Then the best trial of each subject was selected to proceed. An approach is offered to overcome this instability by forcing and educating the brain activities of subjects. The name of this approach is also proposed as over-trial or training effects. According to this view, the classification performance can be improved by increasing the experience and the accumulation of training [45]. Similar evidence was observed as shown in Table 2; experimental setup- 2 and setup- 3 performances between the three setups have the highest performance for ten subjects outside of Subject_3 and Subject_9. Moreover, fewer parameters in experimental setups indicate more degrees of freedom [46]. At this point, the experimental parameters in the GKP-based TMI study are only necessary and basic parameters, as shown in Fig.1. Furthermore, it has been noted that the long cognitive planning time and flexible 
experiments can allow participants for instincts thoughts and goal oriented success in BCI research [45,51]. Therefore, this statement might advance GKP-based TMI in future studies.

\section{CONCLUSION}

This paper aims to develop a novel machine learning approach for a tongue-machine interface using solely glossokinetic potential responses for disabled persons. Compared to other hardware-based studies, GKP-based TMI may provide a natural, reliable, speedy control and communication channel via 1-D simple tongue motions. Then highly exposure of glossokinetic potential responses over the frontal and temporal regions may also help to understand of association between the machine learning algorithms and GKP biosignals. Besides this outcome may cause to design wearable and easy-to-use brain-machine interfaces and tongue-machine interfaces via fewer electrodes. As far as we know, this point and glossokinetic potential responses have been handled for the first time implementing DT and $\mathrm{kNN}$ with mean-absolute value and power spectral density methods. According to our previous research articles, the rule-based algorithm (Decision Tree with Gini Index) is less successful in individual and average results compared to the kernel-based algorithms (Support Vector Machine and Linear Discriminant Analysis) and Neural Network Algorithms (Multi-Layer Neural Network and Probabilistic Neural Network) [55, 56].

Nevertheless, Decision Trees perform well in EEG signals and that these models are easy to understand for the experts due to the rule-based structure [57]. For this reason, Decision Trees are very popular and sometimes selected over a more accurate but less interpretable machine learning algorithms [32]. However, the performance of the Decision Tree can severely be affected by the non-stationary nature of EEGs [57]. Thus, it was observed that voluntary GKP signals generated by tongue contacts were not affected as much as EEG signals via exploring and comparing the potential of the DT and $\mathrm{kNN}$ in required close classification results. Moreover, CNN's performance of the previous study has shown that DT and kNN performances have not achieved as $\mathrm{CNN}$, but highly promising results were provided by these methods [58].

The results of the article indicated that highly acceptable values and investigation of glossokinetic potential responses might demonstrate a novel and promising technique. Therefore GKP-based TMI may be an alternative real-time approach for conventional EEG-based BCIs. Since significant deficiencies arise from the nature of EEG signals, such as low signal-to-noise ratio and internally induced nonstationary mental activities or other external variables. Moreover, GKPs can be compared with the magnetoencephalography signals (MEG) in term of PCA and ICA in the future TMI studies [59-60].

Acknowledgements The authors would like to thank the students of the University of Yozgat Bozok for providing the participation for this research.

\section{Compliance with ethical standards}

Conflicts of interest There is no conflict of interest between the authors.

Ethical approval The Ethical Committee of Sakarya University approved this research. All procedures were performed in accordance with the ethical standards of the institutional and/or national research committee.

Informed consent Informed consent was obtained from all individual participants included in the study.

\section{REFERENCES}

[1] X. Huo, M. Ghovanloo, "Tongue Drive: A wireless tongue-operated means for people with severe disabilities to communicate their intentions", IEEE Comm. Magaz., vol.50, no.10, pp.128-135, 2012.

[2] L.N.S. Andreasen Struijk, "An inductive tongue computer interface for control of computers and assistive devices," IEEE Trans on Biomed Engin., vol. 53, no.12, pp. 2594-2597, 2006

[3] Y. Nam, Q. Zhao, A. Cichocki, S. Choi, "TongueRudder: A Glossokinetic-Potential-Based tongue-machine interface," IEEE Trans. on Bio Engin., vol.59, no.1, pp.290299, 2012.

[4] Y. Nam, B. Koo, A. Cichocki, S. Choi, "GOM-Face: GKP, EOG, and EMG-Based multimodal interface with application to humanoid robot control," IEEE Trans. on Biomed. Engin. vol.61, no.2, pp.453-462, 2014.

[5] Y. Nam, B. Koo, A. Cichocki, S. Choi, "Glossokinetic Potentials for a tongue-machine interface," IEEE Systems, Man, \& Cybernetics Magaz., vol.2, no.1, pp.6-13, 2016.

[6] H. Tang, D.J. Beebe, "An oral tactile interface for blind navigation," IEEE Trans On Neural Sys. and Rehab. Engin., vol.14, no.1, pp.116-123, 2006.

[7] X. Bao, J. Wang, J. Hu, "Method of individual identification based on electroencephalogram analysis," Inter Conf on New Trends in Infor and Ser Sci. pp.390-393 (DOI: 10.1109/NISS.2009.44. 2009).

[8] K.J. Miller, P. Shenoy, M. Nijs, L.B. Sorensen, et.al,. "Beyond the Gamma Band: The role of high-frequency features in movement classification," IEEE Trans. on Biomed. Engin. vol.55, no.5, pp.1634-1637, 2008.

[9] D. Xiao, J. Hu, "Identification of motor imagery EEG signal," Inter Conference on Biomedical Eng and Computer Science, 2010; Wuhan, China.

[10] B. Reuderink, M. Poel, A. Nijholt, "The impact of loss of control on movement BCIs," IEEE Trans on Neural Syst. and Reha. Engin., vol.19, no.6, pp.628-637, 2011.

[11] X. Huo, J. Wang, M. Ghovanloo, “A magneto-inductive sensor based wireless tongue-computer interface," IEEE Trans on Neural Syst. and Reha. Engin., vol.16, no.5, pp.497-504, 2008.

[12] R. Rupp, M. Rohm, M. Schneiders, A. Kreilinger, G.R. 
Müller-Putz. "Functional rehabilitation of the paralyzed upper extremity after spinal cord injury by noninvasive hybrid neuroprostheses," Proceedings of the IEEE, vol.103, no.6, pp.954-968, 2015.

[13] L.M. Alonso-Valerdi, F. Sepulveda, "Development of a simulated living environment platform: Design of BCI assistive software and modelling of a virtual dwelling place," Computer Aided Design, vol,54, pp.39-50, 2014.

[14] X. Huo, J. Wang, M. Ghovanloo, "Using magnetoinductive sensors to detect tongue position in a wireless assistive technology for people with severe disabilities," IEEE Sensor Conf; 2007, Atlanta, USA.

[15] X. Huo, J. Wang, M. Ghovanloo, “A wireless tonguecomputer interface using stereo differential magnetic field measurement," Proceedings of the 29th Ann Inter Conf of the IEEE EMBS Cité Internationale, 2007, Lyon, France.

[16] X. Huo, J. Wang, M. Ghovanloo, "A magnetic wireless tongue-computer interface," Proceed of the 3rd Inter IEEE EMBS Conf on Neural Engineering, 2007, Kohala Coast, Hawaii, USA.

[17] G. Krishnamurthy, M. Ghovanloo, "Tongue Drive: A tongue operated magnetic sensor based wireless assistive technology for people with severe disabilities," IEEE Inter Sym on Circuits and Systems (ISCAS), pp.5551-5554, 2006. [18] R. Vaidyanathan, B. Chung, L. Gupta et.al., "Tonguemovement communication and control concept for handsfree human-machine interfaces," IEEE Trans. on Sys. Man and Cybernetics. vol.37, no.4, pp.533-546, 2007.

[19] R.Vaidyanathan, C.J. James, "Independent component analysis for extraction of critical features from tongue movement ear pressure signals," Proceed of the 29th Ann Inter Conf of the IEEE EMBS Cité Internationale; 2007; Lyon, France.

[20] R. Vaidyanathan, L. Gupta, H. Kook, J. West, “A decision fusion classification architecture for mapping of tongue movements based on aural flow monitoring," Proceed of the IEEE International Conference on Robotics and Automation, 2006; Orlando, Florida.

[21] R. Vaidyanathan, M. Fargues, L. Gupta et.al., "A dualmode human-machine interface for robotic control based on acoustic sensitivity of the aural cavity," IEEE/RAS-EMBS International Conference on Biomedical Robotics and Biomechatronics, BioRob’06, 2006, Pisa, Italy.

[22] R. Vaidyanathan, H. Kook, L. Gupta, J. West, "Parametric and non-parametric signal analysis for mapping air flow in the ear-canal to tongue movements: A new strategy for hands-free human-machine interfaces," IEEE International Conference on Acoustics, Speech, and Signal Processing Proceedings, 2004, Montreal, Canada.

[23] H. Jasper, "The ten twenty electrode system of the international federation," Electro Clin Neuro., vol.10, no.2, pp.370-375, 1958.

[24] M.S. Bascil, A.Y. Tesneli, F. Temurtas, "Spectral feature extraction of EEG signals and pattern recognition during mental tasks of 2-D cursor movements for BCI using SVM and ANN," Australas Phys. Eng. Sci Med. vol. 39, no.3, pp.665-676, 2016.
[25] N. Yalcın, G. Tezel, C. Karakuzu, "Epilepsy diagnosis using artificial neural network learned by PSO," Turk J. Elec. Eng \& Comp. Sci. vol.23,pp.421-432, 2015.

[26] K.D. Desai, M.S. Sankhe, "A Real-Time Fetal ECG Feature Extraction Using Multiscale Discrete Wavelet Transform," 5th Int Conf. on Biomedical Eng. and Infor., pp. 407-412, 2012.

[27] A. Hamad, E.H. Houssein, A.E. Hassanien, A.A. Fahmy, "Feature Extraction of Epilepsy EEG using Discrete Wavelet Transform," 12th Int. Computer Engineering Conf., pp.109-195, 2016.

[28] T.K. Patel, P.C.Panda, S.C. Swain, "Mohanty SK. A Fault Detection Technique in Transmission Line By using Discrete Wavelet Transform," 2nd Int. Conf. on Electrical, Computer and Communication Tech., 2017.

[29] E.J. Rechy-Ramirez, H. Hu, "Bio-signal based control in assistive robots: a survey," Digital Communications and Networks, vol.1, no.2, pp.85-101, 2015.

[30] J.G. Proakis, D.G. Manolakis, "Digital signal processing principles, algorithms and applications," 3rd edn Prentice-Hall, New York [chapter 12]; 1996.

[31] P. Stoica, R. Moses, "Spectral analysis of signals," Prentice Hall International, New York. 2005.

[32] E. Alpaydin, "Introduction to Machine Learning," MIT Press, Cambridge, Massachusetts, Second Edition. 2010.

[33] M. Kavita, M.R. Vargantwar, M.R. Sangita, "Classification of EEG using PCA, ICA and neural network,” Int. J. Eng. Adv. Technol., vol. 1, pp.1-4, 2011.

[34] R. Vigário, J. Särelä, V. Jousmäki, et.al. "Independent component approach to the analysis of EEG and MEG recordings," IEEE Trans. on Biomed. Engin. vol.47, no.5, pp.589-593, 2000.

[35] R.Chai, R.G. Naik, N.T. Nguyen, et.al., "Selecting optimal EEG channels for mental tasks classification: An approach using ICA," IEEE Congress on Evolutionary Computation (CEC), pp.1331-1335, 2016.

[36] B. Şen, M. Peker, "Novel approaches for automated epileptic diagnosis using fcbf selection and classification algorithms," Turk J. Elec. Eng \& Comp. Sci. vol.21, pp.2092-2109, 2013.

[37] R.A. Ramadan, A.V. Vasilakos, "Brain computer interface: control signals review," Neurocomputing. vol.223, pp.26-44, 2017.

[38] B. Obermaier, C. Neuper, C. Guger, G. Pfurtscheller, "Information transfer rate in a five-classes brain-computer interface," IEEE Trans. on Neural Syst. and Reha., vol.9, no. 3, pp.283-288, 2001.

[39] M. Sengelmann, A.K. Engel, A. Maye, "Maximizing information transfer in ssvep-based brain-computer interfaces," IEEE Trans. on Biomedical Engin. vol.64, no.2, pp.381-394, 2017.

[40] B. Wang, C.M. Wong, F. Wan et.al., "Comparison of Different Classification Methods for EEG-Based Brain Computer Interfaces: A Case Study," IEEE Int. Conf on Infor and Automation, Zhuhai/Maca, China, pp.1416-1421, 2009.

[41] K. Gorur, M.S. Bascil, M.R. Bozkurt, F. Temurtas, 
"Classification of Thyroid Data Using Decision Trees, kNN and SVM Methods," International Artificial Intelligence and Data Processing Symposium, IDAP'16, Malatya, Turkey, pp. 130-134, 2016.

[42] Ö. Aydemir, T. Kayıkçıoğlu, "Investigation of the most appropriate mother wavelet for characterizing imaginary EEG signals used in BCI systems," Turk J. Elec. Eng. \& Comp. Sci. vol.24, pp.38-49, 2016.

[43] S. Vanhatalo, J. Voipio, A. Dewaraja, et.al., "Topography and elimination of slow EEG responses related to tongue movements," NeuroImage, vol. 20, pp.1419-1423, 2003.

[44] Y. Nam, K. Bonkon, S. Choi, "Language-related glossokinetic potentials on scalp," IEEE International conference on systems, Man, and Cybernetics, San Diego, USA, 2014.

[45] R. Leeb, F. Lee, C. Keinrath, R. Scherer, et.al., "Braincomputer communication: motivation, aim, and impact of exploring a virtual apartment," IEEE Trans. on Neural Syst. and Reha., vol.15, no.4, pp.473-481, 2007.

[46] V. Jayaram, M. Alamgir, Y. Altun, B. Schölkopf, "Grosse-Wentrup M. Transfer learning in brain-computer interfaces," IEEE Computational Intelligence Magazine, pp.20-31, 2016.

[47] J.C. Kao, S.D. Stavisky, et.al., "Information systems opportunities in brain-machine interface decoders,", Proceedings of the IEEE; vol.102, no.5, pp.666-682, 2014.

[48] A.B. Barreto, A.M. Taberner, L.M. Vicente, "Classification of spatio-temporal EEG readiness potentials towards the development of a brain-computer interface, bringing together education, science and technology," Proceedings of the IEEE, Tampa, USA, 1996.

[49] S.Cerutti, "In the Spotlight: Biomedical signal processing," IEEE Reviews In Biomedical Engin. vol.2, pp.9-11, 2009.

[50] X. Chen, C. He, J. Z .Wang et.al., "An IC-PLS framework for group corticomuscular coupling analysis," IEEE Trans on Biomed Engin. vol.60, no.7, pp.2022-2033, 2013.

[51] J.J. Daly, Y. Fang, et.al., "Prolonged cognitive planning time, elevated cognitive effort, and relationship to coordination and motor control following stroke," IEEE Trans on Neural Syst. and Reha. Engin. vol.14, no.2, pp.168$171,2006$.

[52] Y. Li, C. Guan, J. Qin, "Enhancing feature extraction with sparse component analysis for brain-computer interface," Proceed. of the IEEE Engin. in Med. and Bio. 27th Annual Conference Shanghai, China, 2005.

[53] H.M. Genc, Z Cataltepe, T. Pearson, "A New PCA/ICA based feature selection method," IEEE Signal Processing and Comm. App. 15th (SIU); 2007.

[54] M.J. McKeown, R. Saab, R. Abu-Gharbieh, "A combined independent component analysis (ICA)/ empirical mode decomposition (EMD) method to infer corticomuscular coupling," IEEE Neural Engin Conf Proceed 2nd Int (EMBS), pp.1-8, 2005.

[55] K. Gorur, M.R. Bozkurt, M.S. Bascil, "Temurtas F. Glossokinetic potential based tongue-machine interface for 1-D extraction," Australasian Physical \& Engineering Sciences in Medicine, vol.41, no.2, pp.379-391, 2018. [56] K. Gorur, M.R. Bozkurt, M.S. Bascil, F. Temurtas, "Glossokinetic Potential Based Tongue-Machine Interface For 1-D Extraction Using Neural Networks," Biocybernetics And Biomedical Engineering. Vol.38, No.3, pp.745-759, 2018.

[57] V.Schetinin, C. Maple, "A Bayesian Model Averaging Methodology For Detecting Eeg," 15th International Conference On Digital Signal Processing, pp. 499-502, 2007.

[58] K. Gorur, M.R. Bozkurt, M.S. Bascil, F. Temurtas," GKP Signal Processing Using Deep CNN and SVM for Tongue-Machine Interface", Traitement du Signal, Vol:36, No:4, pp.319-329, 2019.

[59] O.Cetin, F. Temurtas, "Classification of Magnetoencephalography Signals Regarding Visual Stimuli by Generalized Regression Neural Network," Dicle Med J.Vol.46, No.1, pp.19-25, 2019.

[60] K. Gorur, M.R. Bozkurt, M.S. Bascil, F. Temurtas,"Comparative Evaluation for PCA and ICA on Tongue-Machine Interface Using Glossokinetic Potential Responses", Celal Bayar Üniversitesi Fen Bilimleri Dergisi, Vol:16, No:1, pp.35-46, 2020. 